\title{
ELECTORAL AND WELFARE CONSEQUENCES OF POLITICAL MANIPULATION OF THE ECONOMY
}

\author{
William R. KEECH* \\ The University of North Carolina at Chapel Hill, Chapel Hill, NC 27514, USA \\ Carl P. SIMON* \\ The University of Michigan, Ann Arbor, MI 48109, USA
}

Received February 1984, final version received November 1984

This paper examines the long-term electoral and welfare consequences of repeated strategies whereby a political office-holder induces cycles in economic variables to maximize his chances of re-election. Unlike other studies of political business cycles, we focus on questions of the desirability of these cyclical patterns and on the long-run properties of these political economic models. Noting that the welfare costs of vote maximizing in a single term extend beyond that term, we examine in detail the properties of the 'long-run equilibrium path' to which such cycles converge. If the economy starts above this path, vote maximizing can lead to increased social welfare and vote margins. However, if the economy starts below this path, vote-maximizing in the present can cause reduced votes and electoral defeat in subsequent terms. This possibility should lead a far-sighted, enlightened politician or political party to eschew vote-maximizing tactics and the political business cycles which accompany them and thus can help explain why empirical studies have not found convincing evidence of the existence of such cycles. This paper also quantifies the dependence of this long-run equilibrium path on the important political and economic parameters of the model.

\section{Introduction}

An elected official has to reconcile a number of different objectives as he carries out the duties of his office. These objectives may include the general welfare of society, the welfare of various social and economic groups, the electoral success of his party, and his own re-election. A central question in the study of representative government is how politicians reconcile these possibly conflicting goals, and what the consequences of alternative strategies are.

In this paper we will examine in detail a model of a special case of this problem. We will present conceptualizations of general social welfare and of votes as similar but distinct functions of economic variables under the control of an elected official. Our purpose is to assess the consequences of a

*We acknowledge the support of NSF Grant no. 8006562. 
strategy of maximizing votes in the next election on subsequent re-election prospects and on social welfare.

In the model we examine, the politician who chooses to maximize votes introduces cyclical patterns in inflation and unemployment with phases determined by the periods between elections. The possibility that political motivations might generate cyclical patterns in economic variables has been suggested by a number of recent authors, and it has been widely discussed in recent economics and political science literature. Most of this literature has been empirical, focusing on the question of whether such 'political business

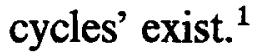

More theoretical questions such as that of the desirability of the cyclical patterns and the long-run properties of these political economic models have received relatively little attention since Nordhaus (1975) presented the basic modern formulation of the rationale behind political business cycles. ${ }^{2}$ Nordhaus suggested that vote-maximizing may involve welfare costs for society as a whole. We will show that while this may be the case, it is not the whole story even within his model. For example, under certain circumstances, policies chosen to maximize the probability of re-election may actually improve social welfare. Moreover, in the more likely situation that such strategies cause welfare loss, the costs of such behavior are limited in two ways. The welfare loss possibilities may be bounded by a long-run equilibrium path; and whether or not they are so bounded, long-run deterioration in social welfare will lead to long-run deterioration in electoral prospects. In this case, a rational and farsighted politician may disdain the political business cycles which are induced by short-run vote maximizing since such cycles may eventually make re-election impossible. Vote maximizing may be at odds with welfare maximizing in the short run in this model, but it cannot lead to an indefinitely increasing welfare loss.

Although the Nordhaus model is only one of several ways in which political motivations can induce cyclic patterns in economic variables, it will be our vehicle of analysis for several reasons. It is the most theoretically sophisticated and mathematically elegant model of the possibility of a conflict between political incentives and public well-being. Among its achievements are the conceptualization of distinct voting and welfare functions that can be optimized subject to economic constraints, and a mathematical solution to the problem of choosing the time-path of a political control variable that maximizes votes. Yet while Nordhaus provides a basis

\footnotetext{
${ }^{1}$ Nordhaus (1957), Tufte (1978) and Maloney and Smirlock (1981) find evidence supportive of the standard political business cycle hypothesis. McCallum (1978), Golden and Poterba (1980), Beck (1982) and Monroe (1983) produce evidence which is not supportive. Paldam $(1979,1981)$ finds cyclical patterns that follow electoral periods, but they do not follow the patterns predicted by the theory we are considering here, and he presents an alternative explanation.

${ }^{2}$ See, however, Lindbeck (1976), and Tufte (1978).
} 
for the analysis of the welfare consequences of selfish and narrow political motivation, his own answer to this question is, as we will show, unsatisfactory and incomplete.

\section{The Nordhaus model}

Nordhaus (1975) conceptualizes both votes and welfare as functions of inflation, $\pi$, and unemployment, $u$, each of which is a 'bad' to be avoided. In fact, for any fixed moment of time, he takes the aggregate voting function $g(u, \pi)$ as a reasonable candidate for a social welfare function. He distinguishes between the voting and welfare functions, not by the sign on inflation or unemployment or even by the marginal rate of substitution between them, but by the time period over which they are evaluated and the method of weighting different times within those periods. Welfare is evaluated across infinite time and is discounted at a rate, $\rho$, in order to weigh present welfare more heavily than future welfare. On the other hand, votes are evaluated across finite electoral periods, and time is discounted backwards rather than forwards, in order to model the fact that voters seem to weigh the recent past more heavily than the distant past in their evaluation of incumbent administrations. While such a voting function is too simple to represent the state of the art in empirical work, its basic features are compatible with that work, which indicates that voters are influenced by a retrospective evaluation of the economic performance of the incumbent administration in which they weigh the recent past more heavily than the earlier part of the present term. ${ }^{3}$

Thus, while the Nordhaus welfare and vote functions are simplifications of reality, they represent well one possible disjuncture between political goals and public well-being. In their general form, the functions are:

$$
\begin{aligned}
& W=\int_{0}^{\infty} g(u, \pi) \mathrm{e}^{-\rho t} \mathrm{~d} t, \\
& V=\int_{0}^{\theta} g(u, \pi) \mathrm{e}^{\mu t} \mathrm{~d} t,
\end{aligned}
$$

where $W$ stands for social welfare, $V$ for aggregate votes, $\theta$ for the length of the electoral period, $\mu$ for the rate of decay of voters' memories, and $g(u, \pi)$ is an aggregate, instantaneous voting function.

To complete this model, Nordhaus includes the now familiar intertemporal interdependence between $u$ and $\pi$, modeled as an expectational Phillips curve; i.e., inflation is a decreasing function of unemployment and an increasing function of expected inflation:

$$
\pi=f(u)+\lambda v, \quad f^{\prime}(u)<0,
$$

${ }^{3}$ See, for example, Fair (1978), Hibbs (1982) and Kramer (1971). 


$$
\dot{v}=\gamma(\pi-v)
$$

where $v$ is expected inflation, $\gamma$ represents the speed of adjustment of expected inflation, and $\lambda$ measures the impact of expected inflation on actual inflation $(0 \leqq \lambda \leqq 1)$. Unemployment, the control variable, can affect inflation directly $(\partial \pi / \partial u<0)$ and indirectly (through $\dot{v}$ when $\pi \neq v)$. The indirect component takes time, as indicated by (4), and as a result the full inflationary or deflationary consequences of present policy may be delayed. For example, unsustainably low combinations of unemployment and inflation at election time can be created by deflation early in the electoral term and may lead to higher inflation for subsequent terms.

The central feature of the Nordhaus model is that politicians might manipulate the timing of economic outcomes to maximize their vote at the next election. Since the vote function and the welfare function are different, such behavior can lead to less than maximal social welfare.

\section{The limitations of Nordhaus's answer to the welfare consequences question}

Nordhaus's own provocative answer to the question of the welfare consequences of political business cycles is that:

'under conditions where voting is an appropriate mechanism for social choice, democratic systems will choose a policy on the long-run trade-off [between inflation and unemployment] that has lower unemployment and higher inflation than is optimal.' [Nordhaus (1975, p. 178).]

Along with the rationale for the existence of a politically-induced business cycle, it is this principle which subsequent analysts regard as the major conclusion of Nordhaus's analysis. ${ }^{4}$ We find this conclusion unsatisfactory for a number of reasons: it is the answer to the wrong mathematical question; it is not necessarily correct; and it fails to distinguish between welfare costs within a term in which votes are maximized and the costs of such maximization in subsequent terms.

Nordhaus derives his conclusion through a consideration of the problem of maximizing the welfare function (1) subject to economic constraints (3) and (4). Without providing any motivation for doing so, he asks only what the system would look like in equilibrium, when all time derivatives are equal to zero. There is no reason to assume that the system will reach such an equilibrium.

Nordhaus finds that when it 'does', 'the democratic outcome corresponds to the policy which was found ... to be purely myopic' $(1975$, p. 179), i.e., where $\rho=\infty$ in (1). He shows that such an equilibrium point has more

\footnotetext{
${ }^{4}$ See, for example, Frey and Ramser (1976), Lächler (1978), MacRae (1977) and Keech (1980).
} 
inflation and less unemployment than does the social welfare maximizing equilibrium corresponding to a finite $\rho$.

However, in the next section of this article, Nordhaus $(1975$, p. 182), introduces what he considers 'a more realistic continuous model', in which he uses the vote function (2) and the memory parameter $\mu$. Once again he uses dynamic optimization techniques. However, this time he does not settle for the undefended static equilibrium solution, but rather uses the usual transversality conditions of control theory to derive a dynamic solution in which the policy-maker sets unemployment at a high level at the beginning of his term and lets it drop monotonically during the term.

Having introduced and worked with this much more realistic model in which he carefully and appropriately uses the techniques of optimal control theory, Nordhaus does not go back to examine how his conclusion about welfare consequences would fit into this more relevant dynamic model. Nor does he attempt to relate the political business cycle paths in his dynamic model to the unmotivated steady-state analysis which he used to derive his optimality conclusion.

We show in appendix $\mathrm{D}$ that when one works with the more realistic dynamic 'business cycle' paths, Nordhaus's principle need not hold. Specifically, we provide an example in Nordhaus's own framework which shows that for certain parameter values each point along a sequence of votemaximizing paths computed using Nordhaus's more dynamic solution, can have more social welfare than the steady state welfare maximizer upon which Nordhaus bases his principle. Furthermore, the inflation rate is lower on the vote-maximizing path than it is at the welfare-maximizing steady state. In the remainder of this paper, we use the more realistic dynamic model to examine the long-run consequences of vote-maximizing strategies both on the reelection chances of the office holder and on the welfare of society as a whole.

\section{Analyzing the welfare consequences of vote maximizing with Nordhaus model}

A first cut at measuring the welfare consequences of vote maximizing might involve assessment of a single term. To do so, one can evaluate both integrals in (1) and (2) from 0 to $\theta$, i.e., over a single electoral period. In this case, (1) and (2) will differ only in their weighting parameters, $-\rho$ and $\mu$. Assuming that $\rho$ is small over a single electoral period, one finds that the welfare costs in that period depend heavily on $\mu$, the parameter representing voters' forgetfulness. Forgetful or present-oriented voters can permit early term high unemployment, which is costly to welfare but can actually enhance the vote for incumbents. Empirical studies by Hibbs (1982) and Fair (1978) suggest that $\mu$ is quite large, certainly large enough to permit a substantial cycle and substantial welfare loss if such a cycle were economically possible and if politicians thought they could get away with it. 
However, the welfare costs of vote maximizing in a single term may extend beyond that term. For example, low terminal values of unemployment may create inflationary expectations in the next term. Even if the incumbent maximized welfare instead of votes in the next term, the feasible values of welfare might be reduced because of the residue of inflationary expectations from the vote maximizing in the previous term.

Thus the welfare costs of vote maximizing in any single term must take into account the effects on welfare in subsequent terms, even if votes are not maximized repeatedly term after term. Since the costs within any term in which votes are maximized are so heavily dependent on the size of $\mu$, the subtler question regards the spillover of residual costs onto the next term. Accordingly, our method of assessing welfare consequences will focus on the consequences after a term in which votes are maximized. These effects are especially important because they are the ones that cannot be eliminated simply by changing the political decision rule in the next term and maximizing welfare. These delayed costs represent both the nature of any trends associated with repeated vote maximizing, and the residual problems that past vote maximizers leave for subsequent welfare maximizers. ${ }^{5}$

\section{Lasting consequences of politically induced business cycles}

Recall that Nordhaus's dynamic model involved maximizing an aggregate voting function $V$ as in (2) with the economic variables constrained by the expectational Phillips curve eqs. (3) and (4). Since expected inflation, $v$, is the state variable in this model, the one variable which politicians cannot directly control, one can uniquely determine a solution to this control theory problem once one sets the initial value of expected inflation, $v(0){ }^{6}$ The solution will include optimal values of $u(t), \pi(t)$ and $v(t)$ for $0 \leqq t \leqq \theta$.

In studying long-run behavior, it is natural to assume that the initial expected inflation $v_{2}(0)$ for the second term equals the terminal value of expected inflation $v_{1}(\theta)$ for the first term. In other words, we assume that the state variable $v(t)$ varies continuously over time - even during changes in

\footnotetext{
${ }^{5}$ Note that our method of assessing welfare consequences of vote maximizing is not dependent on any assumption that all politicians maximize votes, or that any politicians do so all the time. There are many reasons why vote maximizing may be undertaken only from time to time. Incumbents may do so only when they are insecure about their re-election prospects and expect to lose otherwise. Some incumbents may choose not to maximize votes because their ethics or their ideology prohibits doing so. Or incumbents may feel that they can 'fool' voters with this sort of manipulation of the timing of economic outcomes once, or maybe even occasionally, but not regularly, especially when the opposition party or the press can call voters' attention to a pattern. Or, as MacRae (1977) suggested, some administrations may think voters are too sophisticated or 'strategic' to allow such manipulation, and act accordingly. Our analysis shows the nature of the lasting consequences of either intermittent or regular vote maximizing.

${ }^{6}$ We assume that $f(u)$ and $-g(u, \pi)$ are concave in $u$ so that standard existence theorems for solutions of optimal control problems apply.
} 
administration. Thus, once we have determined the optimal $u_{i}(t), \pi_{i}(t)$, and $v_{i}(t)$ for one term (the $i$ th) and agree that $v_{i}(\theta)=v_{i+1}(0)$ for the next term, optimal control theory then determines the optimal $u_{i+1}(t), \pi_{i+1}(t)$, and $v_{i+1}(t)(0 \leqq t \leqq \theta)$ for the succeeding term.

Our analysis of the long-run consequences of vote maximizing will be presented in terms of the difference, $D_{i}$, between expected inflation at the beginning of term $i$ and the end of term $i$,

$$
D_{i}=v_{i}(\theta)-v_{i}(0)
$$

When $D$ is positive, there is a trend of welfare loss across the term, because inflation in the next term will be higher unless there is a compensating increase in unemployment. Similarly, when $D$ is negative, there is a trend of welfare gain. When $D$ is zero, there is no welfare trend, and all welfare costs of vote maximizing are felt within the term. In this case, $v_{i}(0)=v_{i}(\theta)=v_{i+1}(0)$. Since $v_{i}(0)$ determines the solution paths of our dynamic optimization problem, when $D_{i}=0$, the optimal $u(t), \pi(t)$, and $v(t)$ paths for the $i$ th term will also be the optimal paths for the $(i+1)$ st term and for every term thereafter. Consequently, it is natural to define a long-run equilibrium path (LREP) as one in which the optimal $u_{i}(t), \pi_{i}(t)$, and $v_{i}(t)$ are independent of $i$. By the above discussion, such a path is characterized by $D_{1}=0$.

To understand the lasting welfare consequences of vote maximizing, we need to know the sign of $D$ for any initial condition. We also need to know whether this sign stays the same after repeated terms of vote maximizing and whether there is convergence to a $L R E P$, where $D=0$. If such a $L R E P$ exists, we need to know whether it is stable, so that if the system is disturbed in any direction, vote maximizing behavior will return it to the same equilibrium path.

To keep the analysis straightforward, we will look at three basic cases in which we study special functional forms for $f(u)$ and $g(u, \pi)$ : (1) Nordhaus's quadratic-linear $g$ with $\lambda<1$, (2) the same $g$ with $\lambda=1$, and (3) MacRae's quadratic-quadratic $g$ with $\lambda=1$. Our mathematical analysis will be presented in the appendices and summarized in the text. The results will also be presented graphically in phase portraits in unemployment-expected inflation space, i.e., $u-v$ space. These diagrams show the movement of expected inflation that is associated with the vote-maximizing unemployment paths from any initial condition $v_{i}(0)$. In fact, the analysis in our phase portraits works for more general $f(u)$ and $g(u, \pi)$, so that most of our conclusions hold in the more general setting.

We will show that $D_{i}$ has the same sign for all $i$ and that the system tends to a stable $L R E P$ as $i$ increases (except in one special case where $\pi_{i}$ and $v_{i}$ increase without bound). We will study the election and welfare results along the $L R E P$ and will describe how the LREP and its welfare values depend 
on the parameters of the problem, such as $\mu, \rho, \lambda$, and $\theta$. At the end, we will summarize the implications of these analytical results on the electoral and welfare consequences of vote-maximizing strategies.

\subsection{Case one: Nordhaus' functional forms: $\lambda<1$}

We first address these questions for the special functional forms which Nordhaus uses in his paper: the linear Phillips curve function $f(u)=\alpha_{0}-\alpha_{1} u$ in (3) and the quadratic-linear voting function $g(u, \pi)=-u^{2}-\beta \pi$ in (2). As shown in Appendix A, when this quadratic-linear objective function is used, the vote-maximizing path of unemployment $u(t)$ is independent of inflation and expected inflation. In particular, $u(0)$ and $u(\theta)$ depend only on the parameters of the model and not on the initial condition $v(0)$. In terms of our phase diagrams in $u-v$ space, all unemployment paths start on the vertical line $u=u(0)$ and end on the vertical line $u=u(\theta) \equiv \frac{1}{2} \alpha_{1} \beta$, as illustrated in fig. 1 .

(a) $\lambda<1$
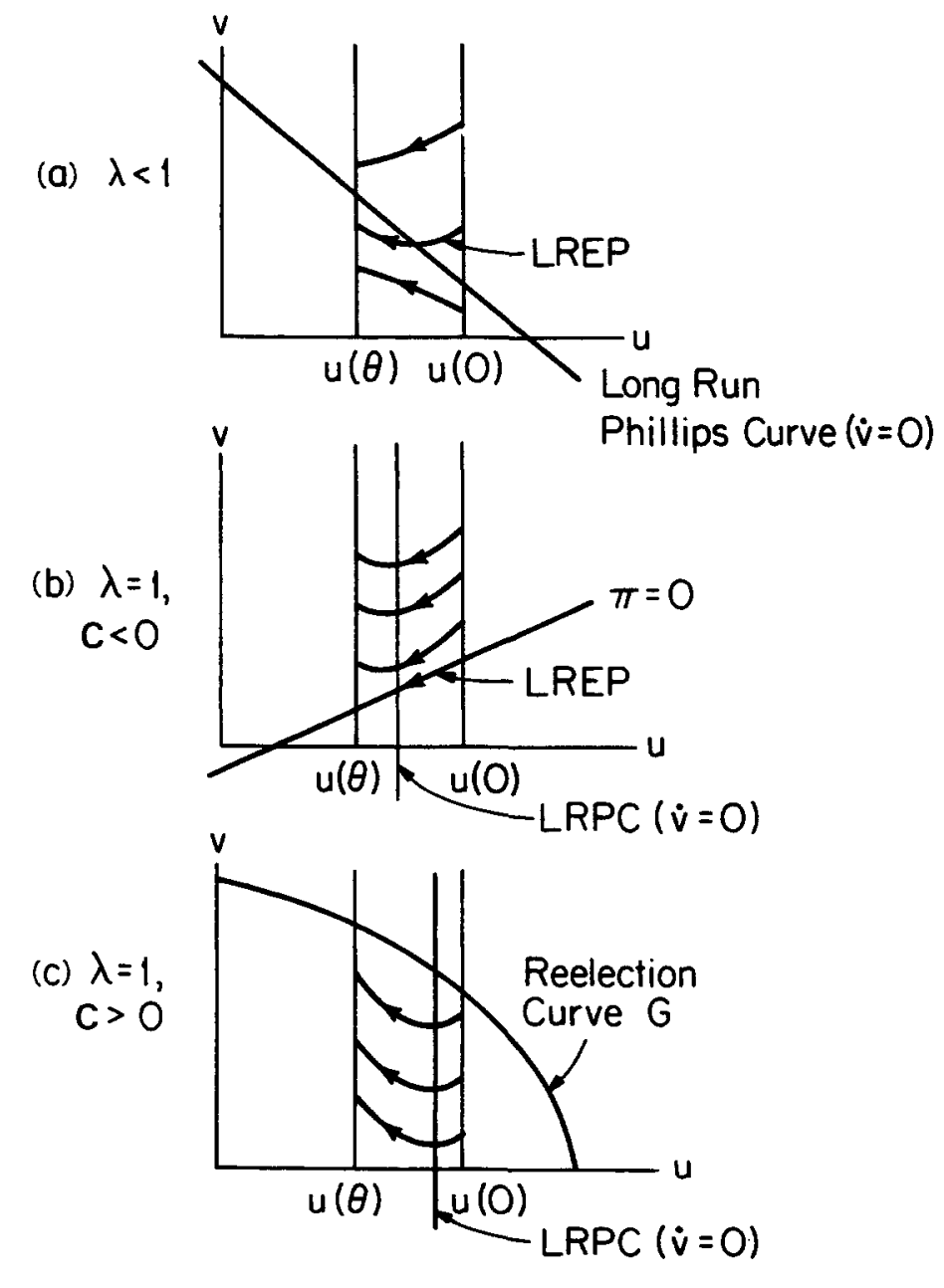

Fig. 1. Phase portraits for Nordhaus' functional forms. 
The corresponding path of inflation depends both on $v(0)$ and on the parameters of the model, especially the size of the coefficient $\lambda$ on expected inflation in (3). This coefficient determines the slope of the long-run Phillips curve, the set of points where inflation is equal to expected inflation and therefore stable. By (4), this curve has the equation $\dot{v}=0$. To its left, inflation is greater than expected inflation and the latter rises; to its right, the reverse holds and expected inflation falls.

When $\lambda<1$, as in fig. 1(a), the long-run Phillips curve is a negatively sloped line which must cut through the $u(0)$ to $u(\theta)$ strip that defines the unemployment path. By the argument of the previous paragraph, political business cycle paths which start above the long-run Phillips curve will move down toward it, while paths which start below the long-run Phillips curve will move up toward it. There is always a unique LREP which crosses the long-run Phillips curve in the $u(0)$ to $u(\theta)$ strip and toward which all the vote-maximizing paths will tend. ${ }^{7}$

The same point can be made mathematically. A long-run equilibrium path of the control variable is one in which $D$ in $(5)$ is zero, or $v(\theta)=v(0)$. As shown in appendix B for the situation under discussion, $v(\theta)$ depends linearly on $v(0)$,

$$
v(\theta)=\mathrm{e}^{-\gamma(1-\lambda) \theta} v(0)+C,
$$

where $C$ depends on all the parameters of the model except $v(0)$. For $0<\lambda<1$, the slope of this line is $\mathrm{e}^{-\gamma(1-\lambda) \theta}$, which lies between 0 and 1 . If one considers (6) as a linear difference equation for $v(\theta)$, the fact that the slope lies between 0 and 1 means not only that there is a unique equilibrium but also that the process converges to this equilibrium from any starting point. Consequently, repeated terms of voting-maximizing politicians would send the economy converging toward a unique and well-defined long-run equilibrium business cycle (LREP). This convergence becomes much more rapid as term length $\theta$ gets longer, as the rate of adjustment of expected inflation $\gamma$ gets larger, or as the coefficient $\lambda$ of expected inflation in (3) gets smaller.

Under the circumstances of Case one, the trend of welfare consequences associated with vote maximizing depends on initial expected inflation. If that initial condition [i.e., the inherited rate of expected inflation, $v(0)$ ] is above the $L R E P$, vote maximizing will lead to improving values of welfare across terms. The reverse is the case if the initial condition is below. In either case, the equilibrium welfare value is that associated with the LREP.

The long term re-election chances of the vote-maximizing incumbent depend on the position of the $L R E P$ relative to the level curve $G$ of $g$ which represents 50 percent of the vote. We have drawn the level curve $G$ in fig.

\footnotetext{
${ }^{7}$ Since $v(0)=v(\theta)$ for a $L R E P$, there exists a $t_{1}$ in $(0, \theta)$ for which $v\left(t_{1}\right)=0$ by the mean value theorem. But $(v=0)$ is the equation for the long-run Phillips curve. Hence, the $L R E P$ must cross the long-run Phillips curve in $u-v$ phase space.
} 
1(c). We call the region below $G$ the 're-election region'. If the LREP ends above the level curve $G$, i.e., outside the re-election region, term by term vote-maximizing strategies will eventually lead to a situation where reelection is impossible. In appendix B, we discuss in detail how the position of the LREP depends on the parameters of the problem. In summary, increases in $\alpha_{1}, \beta, \gamma, \mu$ and $\theta$ and decreases in $\alpha_{0}$ yield LREPs which are closer to the origin in $u-v$ space and thus have a higher level of social welfare and a greater chance of falling within the re-election region.

\subsection{Case two: Nordhaus' functional forms: $\lambda=1$}

Macroeconomists of various theoretical persuasions suggest that $\lambda$ is very close to 1 , if not identically 1 , in inflation models for the contemporary era. As $\lambda$ approaches 1 , the slope of the long-run Phillips curve becomes infinite, i.e., the curve becomes vertical. When $\lambda=1$, the long-run Phillips curve is a vertical line which need not intersect the $u(0)-u(\theta)$ strip. Then succeeding vote-maximizing paths move monotonically up or down in the strip as illustrated in figs. 1(b) and 1(c).

This movement can also be seen analytically by examining the difference eq. (6). When $\lambda=1$, the coefficient of $v(0)$ becomes 1 and the difference equation is now a simple translation

$$
v(\theta)=v(0)+C,
$$

which will move continually upwards or downwards depending on whether $C$ is positive or negative.

Given the functional form of Nordhaus' social welfare function (1) and voting function (2), sequences of business cycles which move upwards to $+\infty$ lead to lower and lower social welfare and to a decreasing percentage of votes for the vote maximizer, while the opposite occurs for sequences which move downward to $-\infty$. However, Nordhaus imposes the constraint $\pi \geqq 0$ on his model (among other reasons) to avoid the situations where arbitrarily high rates of disinflation lead to arbitrarily high levels of social welfare. Since $\pi=\alpha_{0}-\alpha_{1} u+\lambda v$ in Nordhaus' framework, business cycle paths cannot fall below the line

$$
\alpha_{0}-\alpha_{1} u+\lambda v=0
$$

in $u-v$ phase portrait space. Thus, instead of going downward to $v=-\infty$, decreasing sequences of business cycles will pile up onto the line (8) as indicated in fig. 1(b). The intersection of line (8) with the $u(0)-u(\theta)$ strip becomes the long-run equilibrium path for this situation. ${ }^{8}$

\footnotetext{
${ }^{8}$ This follows from the maximum principle of control theory or from Arrow's principle of blocked intervals. See, for example, Clark (1976).
} 
Just as Nordhaus' $\pi \geqq 0$ constraint limits the welfare gain, the existence of the 're-election region' implicitly bounds the welfare loss associated with vote maximizing when $C$ is positive. Since the vote and welfare functions have the same arguments, successive cycles which include higher expected inflation will cause higher inflation and therefore fewer votes as well as less welfare. Once one of these increasing cycles ends outside the re-election region, the vote-maximizing incumbent will find himself in a position where the maximum attainable number of votes will not be enough to win re-election. Ironically, vote maximizing in the present can cause reduced votes and electoral defeat in subsequent terms. This possibility should lead a farsighted, enlightened politician or political party to eschew vote-maximizing tactics and the political business cycles which accompany them.

Whether sequences of political business cycles move up beyond the reelection region or down to $\pi=0$ when $\lambda=1$ depends on the sign of the constant $C$ in eq. (7). As appendix B explains, an increase in $\alpha_{1}, \beta, \gamma, \mu$, or $\theta$, or a decrease in $\alpha_{0}$ or in the natural rate of unemployment increases the likelihood that $C$ will be negative and that welfare or votes will grow during successive political business cycles. Furthermore, the most important comparison is that between the unemployment rate during the cycle and the natural rate of unemployment, $\alpha_{0} / \alpha_{1}$. If the unemployment rate during most or all of the cycle stays above the natural rate, then successive cycles will lead to improved welfare and vote margins. On the other hand, if the votemaximizing politician keeps the unemployment rate below the natural rate for all or most of his term, then successive cycles will lead to diminishing social welfare and vote margins.

\subsection{Case three: The fully quadratic voting function}

As indicated above, the quadratic-linear vote function makes the votemaximizing path of unemployment independent of inflation and expected inflation. That is, no matter what the inflationary situation is, and no matter what the inflationary consequences are, the choice of the control will be exactly the same. This invariant path seems rather implausible from both a theoretical and a practical standpoint.

The fully quadratic objective function $g(u, \pi)=-u^{2}-\beta \pi^{2}$ is more sensible theoretically, although it is less tractible mathematically. ${ }^{9}$ The quadraticlinear form allowed closed form solutions for the $u, v$ and $\pi$ paths. In the fully quadratic case, the differential equations are still linear, as they were for the Nordhaus formulation. However, the eigenvalues for this linear system are complicated irrational expressions of the parameters of the problem, so a

\footnotetext{
${ }^{9} \mathrm{~A}$ fully quadratic functional form is used by MacRae (1977), Maloney and Smirlock (1981), and Chappell and Keech (1983).
} 
closed form solution is no longer illuminating. Still, one can use phase portrait analysis in $u-v$ space to study the properties of the modified system, just as we did with Nordhaus' formulation. We carry out this analysis in appendix $\mathrm{C}$ and summarize its results here.

The exact nature of the dynamics depends on the relative sizes of two of the parameters, $\gamma$ and $\mu$, as explained in appendix $C$, but the essential nature of the results is the same in each case. Accordingly, we have presented a stylized phase portrait in fig. 2 , which represents the central features of our results.

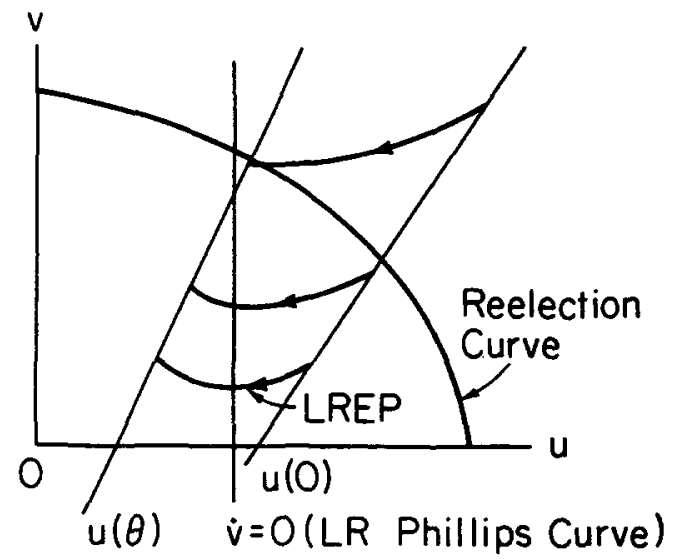

Fig. 2. Phase portrait for fully quadratic objective function.

In the fully quadratic case, the vote-maximizing path of the control variable, $u^{*}(t)$, is still a decreasing function of $t$ for $0 \leqq t \leqq \theta$, which means that politically induced business cycles still exist with this objective function. However, as indicated in our stylized fig. 2, the initial and terminal values of unemployment are no longer invariant with respect to expected inflation, as they were in fig. 1, which represented the quadratic-linear case. Now, the $t=\theta$ and $t=0$ lines are positively sloped in $u-v$ space, implying that higher values of unemployment will be chosen when expected inflation is high. That is, the optimal unemployment path compensates for high inflationary expectations, and is chosen to bring them down towards more desirable values of the objective functions. This formulation makes more sense theoretically than the quadratic-linear alternative, which implies that an optimal unemployment path can cause inflation but is insensitive to it, even though inflation is an argument in the objective function being optimized.

There is a unique long-run equilibrium path for the fully quadratic objective function for any choice of economic and political parameters. Any sequence of cycles which satisfies our natural continuity assumption, $v_{i+1}(0)=v_{i}(\theta)$, will tend to this $L R E P$ as $i$ increases, i.e., the LREP is 'globally stable'. For the rest of this analysis, we will work with the widely accepted hypo- 
thesis that $\lambda=1$. Even in this case, the vertical long-run Phillips curve will still cross the $u(0)$ to $u(\theta)$ region and a unique finite $L R E P$ will exist. ${ }^{10}$

The lasting consequences of vote maximizing now depend on the initial values of expected inflation. If the initial condition lies above the $L R E P$, then $D_{1}$ is negative and the successive cycles will move downward to that path, with voter support and social welfare increasing as the sequence progresses. If the initial position lies below the LREP, then $D_{i}$ is positive, and the successive cycles will move upward to that path, with votes and welfare decreasing.

The position of the LREP now becomes critical. If it lies in the re-election region under the 50 percent contour, then repeated vote maximizers may still be able to retain their office. However, if the economy is initially below the $L R E P$, decreasing vote margins and loss of social welfare could lead voters and candidates to reject the mechanisms of the political business cycle. If the LREP lies beyond the 50 percent vote contour, then we have the same situation as we had when the business cycles marched to infinity in the Nordhaus formulation. Officeholders will eventually be unable to retain their offices if they continue to maximize votes term by term, and if the 50 percent vote contour remains fixed. This fact, together with the diminished social welfare of the voters, should lead to a discontinuation of the vote-maximizing techniques that lead to political business cycles. The system may become selfdefeating in its own terms.

As before, the question of how the economic and political parameters affect the location of the LREP is an important issue. As derived in appendix $C$, the comparative statics are similar to those for Nordhaus' original formulation. Increases in $\alpha_{1}, \beta, \mu$, or $\theta$ or a decrease in $\alpha_{0}$ yield LREPs with higher welfare levels and vote margins. Once again, the higher the natural rate of unemployment, the more likely it is that successive business cycles will lead to shrinking vote margins and welfare levels. ${ }^{11}$

\section{Conclusion}

We have found that repeated vote maximizing leads to two kinds of welfare possibilities and to two kinds of political possibilities. The critical concept is the long-run equilibrium path (LREP) and its position relative to

\footnotetext{
${ }^{10}$ In fact, $v(\theta)$ is still a linear function of $v(0)$ as in (6); its slope always lies between 0 and 1.

${ }^{11}$ As the analysis in appendix $C$ indicates, the conclusions of this section hold for much more general voting functions $g(u, v)$ and inflation-unemployment relationships $f(u)$. In general, the optimal $u(t)$ will be a decreasing function of $t$ for $0 \leqq t \leqq \theta$; its values will depend on the initial condition $v(0)$. Either the business cycles will march off to $\infty$ in $u-v$ space for successive administrations, or (more likely) there will exist at least one LREP and each sequence of business cycles satisfying $v_{i+1}(0)=v_{i}(\theta)$ will tend to some $L R E P$. The long-run welfare consequences of vote maximization in this context will depend heavily on the position of the $L R E P$ relative to the 50 percent isoquant of the vote function, as described earlier in this section.
} 
the 50 percent vote isoquant, or re-election line. We showed that in general stable $L R E P$ s exist, possibly at infinity. In the cases we examined, the $L R E P$ was unique and the sequence of business cycles converged monotonically toward it.

The two kinds of welfare possibilities depend on whether the initial condition is above or below the LREP in unemployment-expected inflation space. If it is above, repeated vote maximizing leads to successive welfare improvements. If it is below, such a process leads to successive welfare losses. In either case, the LREP represents a stable equilibrium with identifiable welfare and political properties.

Whether repeated vote maximizing reaches the $L R E P$ depends on the location of that path relative to the re-election line. If the path is below that line, vote maximizing can be compatible with repeated re-election whether it is associated with welfare improvements or welfare losses. If the $L R E P$ is beyond that line, repeated vote maximizing will sooner or later lead to the ironic situation in which maximizing votes will not secure re-election.

Our comparative statics analysis indicates that the $L R E P$ is more likely to lie outside the re-election region: the higher the natural rate of unemployment, the better voters' memories, the shorter the electoral term length, and the greater the weight that voters give to unemployment relative to inflation. Furthermore, the longer a vote-maximizing politician keeps the unemployment rate below the natural rate, the greater are the chances that his strategy will lead to diminishing social welfare and vote margins across terms.

The Nordhaus model has conceptualized a way to assess the welfare consequences of narrow political motivation. Politicians are assumed to have a great deal of control over the economy, and therefore over votes and welfare. While welfare is defined basically in terms of what voters want, the basis for the disjuncture between what brings votes and what brings welfare is a failure to understand the intertemporal interdependence of inflation and unemployment, and/or a failure to take a long view.

We have shown that in a world so described there are inherent limits on the welfare loss associated with vote maximizing strategies. There are circumstances in which such strategies actually increase social welfare. But even when they lead to welfare losses, the 50 percent vote isoquant provides a natural bound so that at worst, repeated vote maximizing will lead to declining votes across terms and finally to repeated defeats of newly elected incumbents. Thus the worst welfare possibilities are associated with the worst political possibilities.

Recognition of this fact may lead farsighted politicians to eschew term by term vote maximizing because they anticipate its political as well as welfare costs. Indeed this may help explain the failure of many investigators to find evidence of political business cycles. ${ }^{12}$ Repeated vote maximizing may not

${ }^{12}$ See footnote 1. 
maximize welfare as if guided by an invisible hand, but there are limits on the amount of damage it can do.

\section{Appendix A: Nordhaus' formulation of the political business cycle model}

Nordhaus (1975) uses a linear Phillips curve, $f(u)=\alpha_{0}-\alpha_{1} u$, in (3) and a quadratic-linear voting function in (1) and (2), $g(u, \pi)=-u^{2}-\beta \pi$, where $\alpha_{0}$, $\alpha_{1}$, and $\beta$ are positive constants. After applying the maximum principle to the problem of maximizing (2) subject to (3) and (4), he arrives at the linear system of differential equations,

$$
\begin{aligned}
& \dot{u}=A u+B, \\
& \dot{v}=\gamma\left\{\alpha_{0}-\alpha_{1} u-(1-\lambda) v\right\},
\end{aligned}
$$

with boundary conditions: $v(0)=v_{0}$ and $u(\theta)=\frac{1}{2} \alpha_{1} \beta$. Here,

$$
A \equiv \gamma(1-\lambda)-\lambda, \quad B \equiv-\frac{1}{2} \alpha_{1} \beta(\gamma-\mu),
$$

where $\mu$ is the parameter measuring voters' memory in (2) and $\gamma$ is the rate of adjustment of expected inflation to discrepancies between actual and expected inflation in (4). Since (A.1) is a linear equation in $u$ alone, one can easily solve it and its boundary condition to obtain the optimal unemployment rate,

$$
u^{*}(t)=\left(\frac{1}{2} \alpha_{1} \beta+B / A\right) \exp [A(t-\theta)]-B / A .
$$

Note that $u^{*}(t)$ is independent of $v(0)$. Now, plug (A.3) into (A.1) to find the corresponding

$$
\begin{aligned}
& v^{*}(t)=G-F \mathrm{e}^{A(t-\theta)}+\left\{v_{0}-G+F \mathrm{e}^{-A \theta}\right\} \mathrm{e}^{-\gamma(1-\lambda) t}, \text { where } \\
& G=\left(\alpha_{0}+\alpha_{1}(B / A)\right) /(1-\lambda), \\
& F \equiv \alpha_{1} \gamma\left(\frac{1}{2} \alpha_{1} \beta+B / A\right) /[2 \gamma(1-\lambda)-\mu] .
\end{aligned}
$$

Phase portraits of the system (A.1) in $u-v$ space can often give more geometric information about the behavior of the system. There are basically two distinct phase portraits, depending on the signs of the constants $A$ and $B$ in (11). We have drawn these two portraits in figs. 3 and 4: one for $A<0$ and $B<0$ and one for $A>0$ and $B<0$. The portrait for $A<0$ and $B<0$ is similar to the former except that the $\{\dot{u}=0\}$-line lies to the left of the $v$-axis. The situation $A>0$ and $B>0$ cannot occur. 


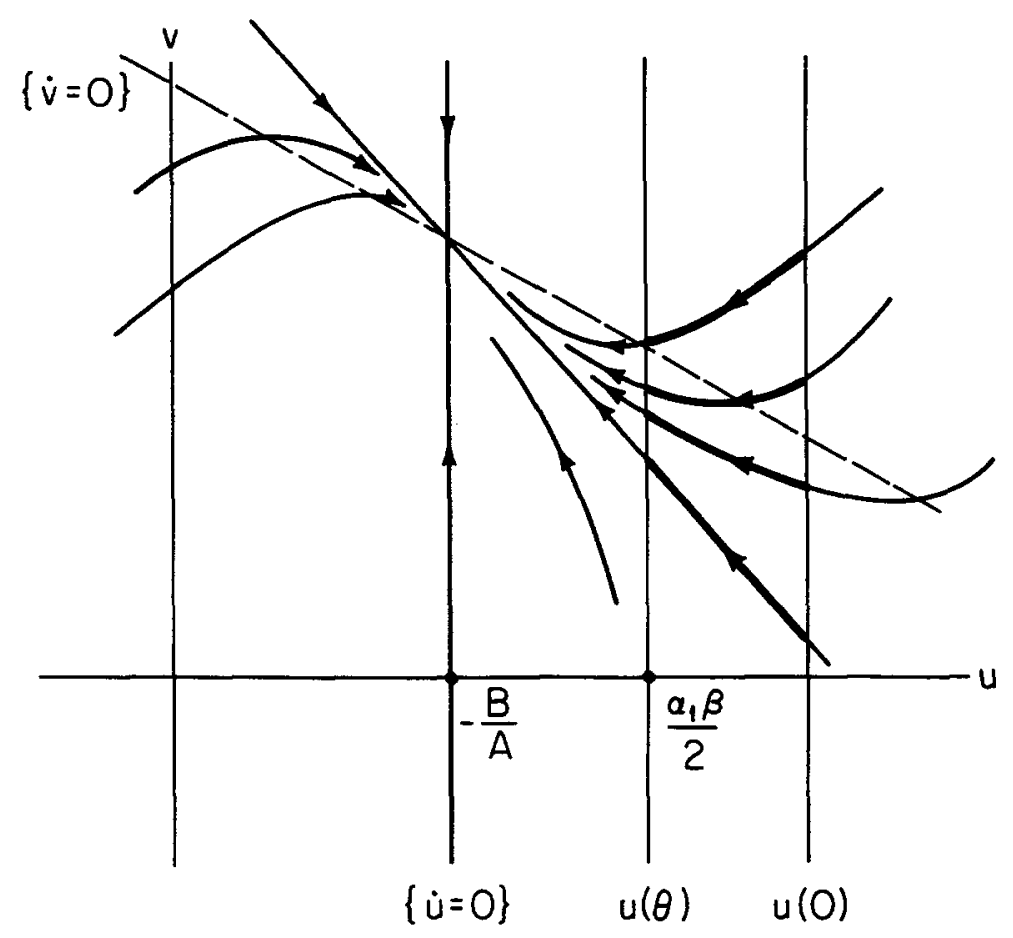

Fig. 3. Phase portrait of (A.1) for $A<0$ and $B>0$.

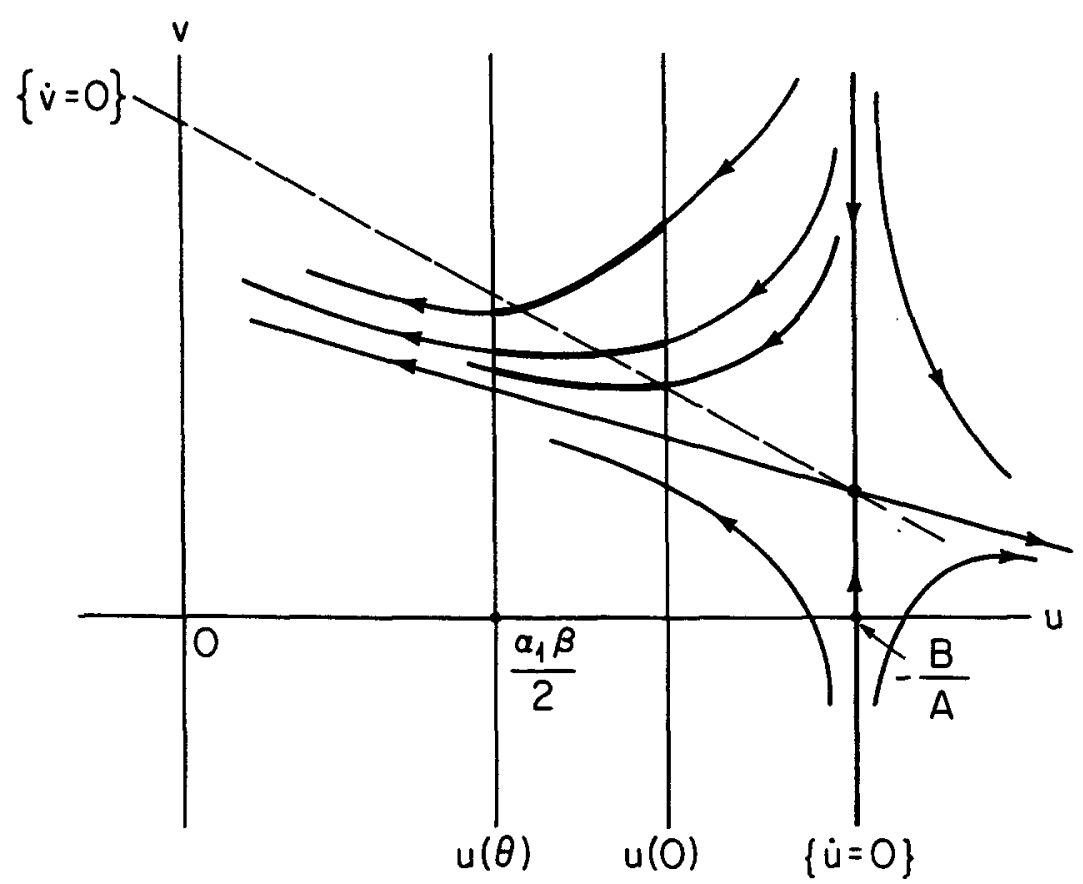

Fig. 4. Phase portrait of (A.1) for $A>0$ and $B<0$. 
There are four important lines in each of these portraits: (1) the vertical $\{\dot{u}=0\}$ line which occurs at $\{u=-B / A\}$ and is an orbit of (A.1), (2) the vertical $u(\theta)$-line which occurs at $u=\frac{1}{2} \alpha_{1} \beta$ and on which all business cycles must end, (3) the vertical $u(0)$-line which by (A.3) occurs at

$$
u(0)=\left(\frac{1}{2} \alpha_{1} \beta+B / A\right) \mathrm{e}^{-A \theta}-B / A
$$

and on which all business cycles begin, and (4) the negatively sloped $\{\dot{v}=0\}$ line whose equation by (A.1b) is

$$
\alpha_{1} u+\gamma(1-\lambda) v=\alpha_{0},
$$

and whose slope is $-\alpha_{1} /(1-\lambda)$. On this last line, which is drawn as a dashed line in the figures, the dynamical system is horizontal. We have also drawn in some typical solution curves. The thicker ones between the $u(0)$ - and $u(\theta)$ lines are examples of political business cycles.

\section{Appendix B: The existence and locations of $L R E P$ s in Nordhaus' model}

To study long-run behavior, one first needs to know how $v(\theta)$ depends on $v(0)$, recalling that this $v(\theta)$ will be the new $v(0)$ for the next term. By plugging $t=\theta$ into (A.4), one obtains

$$
\begin{aligned}
& v(\theta)=\mathrm{e}^{-\gamma(1-\lambda) \theta} v(0)+C_{1}, \quad \text { where } \\
& C_{1} \equiv G\left(1-\mathrm{e}^{-\gamma(1-\lambda) \theta}\right)-F\left(1-\mathrm{e}^{-(A+\gamma(1-\lambda) \theta}\right)
\end{aligned}
$$

does not depend on $v(0)$. Eq. (B.1) is a linear difference equation for $v(\theta)$ with the coefficient of $v(0)$ a constant between 0 and 1 . Therefore, iteration of this difference equation with $v_{i+1}(0)=v_{i}(\theta)$ and $v_{1}(0)$ arbitrary will always converge to its unique steady state solution

$$
\begin{aligned}
v(\theta) & =v(0)=C_{1} /\left(1-\mathrm{e}^{-\gamma(1-\lambda) \theta}\right) \\
& =G-F\left(1-\mathrm{e}^{-(A+\gamma(1-\lambda)) \theta}\right)\left(1-\mathrm{e}^{-\gamma(1-\lambda) \theta}\right)^{-1},
\end{aligned}
$$

where $F$ and $G$ are the constants defined in (A.5).

Having seen that a unique LREP exists, we next determine how its location depends on the economic and political parameters of this model (e.g., $\alpha_{0}, \alpha_{1}, \beta, \gamma, \lambda, \mu, \theta$ ). The direct method for such a study would be to differentiate (B.2) with respect to each of these parameters. Because of the complexity of (B.2), this method is very unwieldy and provides little insight. Instead, we will see how changes in these parameters affect the four principal lines in the phase portraits of figs. 3 and 4. 


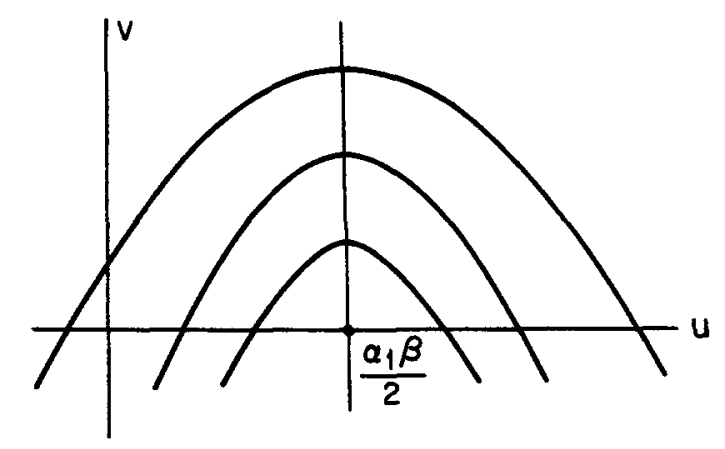

Fig. 5. Level set of Nordhaus' vote/welfare function.

Recall that Nordhaus' vote/welfare function is

$$
\tilde{g}(u, v) \equiv g\left(u, \alpha_{0}-\alpha_{1} u+\lambda v\right)=-u^{2}-\beta\left(\alpha_{0}-\alpha_{1} u+\lambda v\right) .
$$

Its level curves are parabolae, each of whose maximum is on the line $u=\frac{1}{2} \alpha_{1} \beta$. (See fig. 5.) Since this is also the $u(\theta)$-line, all business cycles will lie to the right of this line. Consequently, if a business cycle such as a LREP moves up or to the right, the new path will have lower levels of welfare. Conversely, if such a cycle moves down or to the left, welfare will increase.

Recall that, by our discussion in the text, the $L R E P$ crosses the $\{\dot{v}=0\}$-line in the $u(0)-u(\theta)$ strip, beginning and ending at the same $v$-level. If, for example, the $u(0)$ - or $u(\theta)$-line moves to the right, the $L R E P$ would have to move down. We first rewrite (A.6) as

$$
u(0)=\frac{1}{2} \alpha_{1} \beta \gamma \lambda \theta k(A \theta)+\frac{1}{2} \alpha_{1} \beta,
$$

where $k(x) \equiv\left(1-\mathrm{e}^{-x}\right) / x$ is the function which played a major role in the analysis in Keech and Simon (1983). One checks easily that $k$ is always positive, $k^{\prime}$ is always negative, and that

$$
k(x)+k^{\prime}(x) x=\mathrm{e}^{-x}>0 .
$$

An increase in $\mu, \theta$, or $\gamma$ will affect the $u(0)$-line but not the $u(\theta)$ - or the $\{\dot{v}=0\}$ lines. Using (B.3), one checks

$$
\begin{aligned}
& \partial(u(0)) / \partial \mu=\frac{1}{2} \alpha_{1} \beta \gamma \lambda \theta^{2} k^{\prime}(A \theta)(-1)>0, \\
& \partial(u(0)) / \partial \theta=\frac{1}{2} \alpha_{1} \beta \gamma \lambda\left[k(A \theta)+A \theta k^{\prime}(A \theta)\right]>0 \quad \text { by }(\mathrm{B} .4), \\
& \partial(u(0)) / \partial \gamma=\frac{1}{2} \alpha_{1} \beta \lambda \theta\left[k(A \theta)+\gamma \theta k^{\prime}(A \theta)(1-\lambda)\right],
\end{aligned}
$$

which is positive at least for $\lambda$ in the realistic range near 1 . Consequently, if 
$\mu, \theta$, or $\gamma$ increase, the $u(0)$-line moves to the right and therefore the $L R E P$ moves down (into a region of higher votes and higher social welfare). If $\alpha_{1}$ or $\beta$ increases, a similar analysis shows that both the $u(0)$ - and $u(\theta)$-line move to the right and so that $L R E P$ moves down. In the case of the increase of $\alpha_{1}$, the move is accentuated by an increase in the steepness of the $\{\dot{v}=0\}$-line. If $\alpha_{0}$ increases, the $\{\dot{v}=0\}$ is the only line affected. It moves upwards, bringing the LREP up with it. Since $\alpha_{0} / \alpha_{1}$ is analogous to the natural rate of unemployment in this model, we see that decreases in this rate lead to a lower (and more preferred) LREP.

As mentioned in the text, it is natural to set coefficient $\lambda$ of expected inflation in the unemployment-inflation function in (3) equal to 1 . When this occurs, the $\{\dot{v}=0\}$-line in figs. 3 and 4 (i.e., the long-run Phillips curve) becomes a vertical line which crosses the $u$-axis at the 'natural rate of unemployment', $\alpha_{0} / \alpha_{1}$. Ironically, there is now a wider diversity of phase portraits, depending on (1) the sign of $B$ in (A.2), (2) the sign of $B / \mu-\alpha_{0} / \alpha_{1}$, and (3) the location of the $u(\theta)-u(0)$ strip, e.g., $\frac{1}{2} \alpha_{1} \beta$, relative to $\alpha_{0} / \alpha_{1}$. We have drawn two of the phase portraits corresponding to $B>0$ in figs. 6 and 7 .

To study long-run behavior for $\lambda=1$, we first need to find the optimal $v^{*}(t)$ and then plug in $t=\theta$ to obtain a difference equation for $v(\theta)$ in terms of $v(0)$.

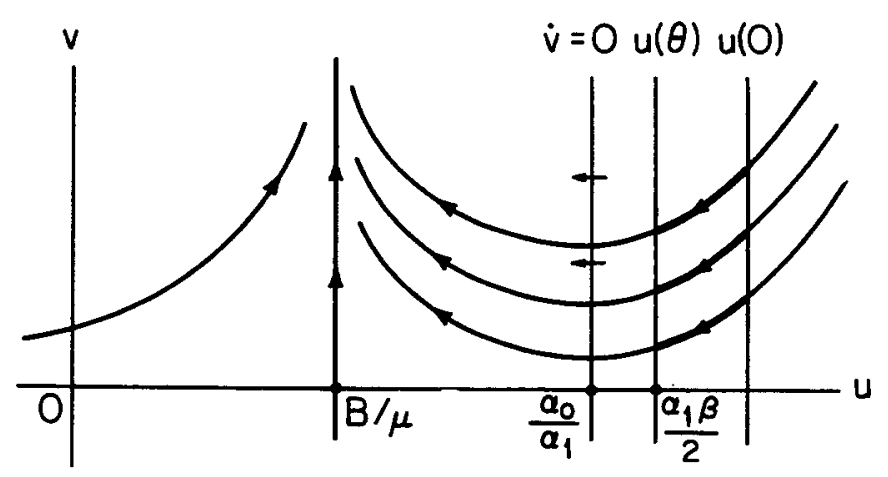

Fig. 6. $B>0, \lambda=1, B / \mu<\alpha_{0} / \alpha_{1}<\frac{1}{2} \alpha_{1} \beta$.

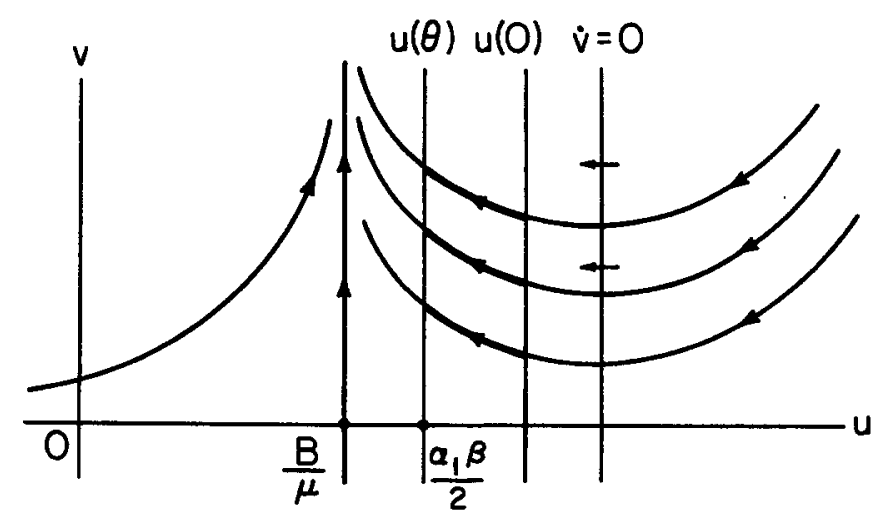

Fig. 7. $B>0, \lambda=1, B / \mu<\frac{1}{2} \alpha_{1} \beta<\alpha_{0} / \alpha_{1}$. 
Straightforward calculations yield

$$
\begin{aligned}
& v(\theta)=v(0)+C_{2}, \quad \text { where } \\
& C_{2} \equiv \alpha_{1} \gamma \theta\left[\left(\alpha_{0} / \alpha_{1}-B / \mu\right)+\left(\frac{1}{2} \alpha_{1} \beta-B / \mu\right)\left(\left(1-\mathrm{e}^{\mu \theta}\right) / \mu \theta\right)\right] .
\end{aligned}
$$

This difference equation for $v(\theta)$ is a simple translation which marches off to $+\infty$ if $C_{2}>0$ and to $-\infty$ if $C_{2}<0$. (In the unlikely case that $C_{2}$ is identically zero, then every business cycle is a $L R E P$; an unstable 'knife-edge' situation which can be destroyed by any change in the parameters.) As we mention in the text, Nordhaus adds the constraint ' $\pi \geqq 0$ ' to avoid the situation where high levels of disinflation lead to high levels of social welfare. As a result, a decreasing sequence of business cycles for $\lambda=1$ will not drop to $-\infty$ but will level off on the line $\pi=\alpha_{0}-\alpha_{1} u+\lambda v=0$.

To complete the welfare study of Nordhaus' model when $\lambda=1$, we need to know what values of the parameters lead to the (ideal) situation of $C_{2}<0$ where decreasing sequences of cycles lead to increases in vote margins and in social welfare and what values lead to the (self-destructing) situation of $C_{2}>0$ where increasing sequences of cycles lead to decreases in vote margins and in social welfare.

Once again, there are two approaches. We can work with the analytic expression (B.6) and find sufficient conditions on the parameters that lead to positive or negative $C_{2}$. One checks easily that

$$
\frac{1}{2} \alpha_{1} \beta-B / \mu>0 \text { and }\left(1-\mathrm{e}^{\mu \theta}\right) / \mu \theta<0,
$$

so the sign of $C_{2}$ depends on the sign and size of $\left(\alpha_{0} / \alpha_{1}-B / \mu\right)$. Alternatively, we can work with the phase diagrams as we did earlier in the appendix for $\lambda<1$. Either method yields the following two conditions: if

$$
\begin{aligned}
& \alpha_{0} / \alpha_{1} \leqq \frac{1}{2} \alpha_{1} \beta=u(\theta) \text { then } C_{2}<0, \text { if } \\
& \alpha_{0} / \alpha_{1} \geqq \frac{1}{2} \alpha_{1} \beta\left(1+\gamma \theta\left(\left(\mathrm{e}^{\mu \theta}-1\right) / \mu \theta\right)\right)=u(0) \text { then } C_{2}>0 .
\end{aligned}
$$

One can draw two types of conclusions from conditions (B.7). First, the comparative statics are similar to those described above for $\lambda<1$. Increases in $\alpha_{1}, \beta, \gamma, \mu$, or $\theta$ or a decrease in $\alpha_{0}$ increase the likelihood that $C_{2}$ will be negative and that welfare and votes will grow during successive political business cycles. Second, the most important comparison is that between the unemployment rate during the cycles and the natural unemployment rate $\alpha_{0} / \alpha_{1}$. If the unemployment rate during most or all the cycle stays above the natural rate, then successive cycles will lead to improved welfare and vote margins. On the other hand, if the vote-maximizing politician keeps the 
unemployment rate below the natural rate for all or most of his term, then successive cycles will lead to diminishing social welfare and vote margins and eventually to a no-win situation.

\section{Appendix C: Analysis with a pure quadratic vote function}

In this appendix, we will study the problem of maximizing (2) subject to (3) and (4) with the vote function in (2) a pure quadratic,

$$
g(u, \pi)=-u^{2}-\beta \pi^{2}
$$

Applying the maximum principle of optimal control theory just as Nordhaus (1975) did, we find that the optimal paths $u(t)$ and $v(t)$ must satisfy the linear system

$$
\begin{aligned}
& \dot{u}=A u-K \lambda[A+\gamma] v-\alpha_{0} A K, \\
& \dot{v}=-\alpha_{1} \gamma u-\gamma(1-\lambda) v+\alpha_{0} \gamma
\end{aligned}
$$

where $A$ is still $(1-\lambda) \gamma-\mu$ and $K \equiv \alpha_{1} \beta /\left(1+\alpha_{1}^{2} \beta\right)$. The transversality condition $\phi(\theta)=0$ becomes

$$
u(\theta)-K v(\theta)=\alpha_{0} K
$$

One notices from (C.2) that $u(\theta)$ now depends on $v(\theta)$ and therefore on $v(0)$, unlike the situation for Nordhaus' vote function.

To keep the mathematics tractable, we will restrict ourselves to the case $\lambda=1$ for the rest of this appendix. The system (C.1) now becomes

$$
\begin{aligned}
& \dot{u}=-\mu \mu-K[\gamma-\mu] v+\alpha_{0} \mu K, \\
& \dot{v}=-\alpha_{1} \gamma u+\alpha_{0} \gamma .
\end{aligned}
$$

There are three different phase portraits, depending on whether $\gamma-\mu$ is positive, zero, or negative. We have drawn two of these portraits in figs. 8 and 9. There are three important lines in each figure: (1) the line $\{\dot{u}=0\}$ whose $u$-intercept is $\alpha_{0} K$ and whose slope is $\mu / K(\mu-\gamma)$, (2) the vertical line $\{\dot{v}=0\}$, whose $u$-intercept is $\alpha_{0} / \alpha_{1}$, and (3) the $\{t=\theta\}$-line give by (C.2), whose $u$-intercept is also $\alpha_{0} K$ and whose slope is $1 / K>0$. Note that $0<\alpha_{0} K<\alpha_{0} / \alpha_{1}$.

The business cycle trajectory [solution to (C.1) and (C.2)] must end on the $\{t=\theta\}$-line in each of these portraits and must satisfy $v(\theta)>0$ by (C.2). To find the solution for a given $v(0)$, find the trajectory in the proper phase portrait 


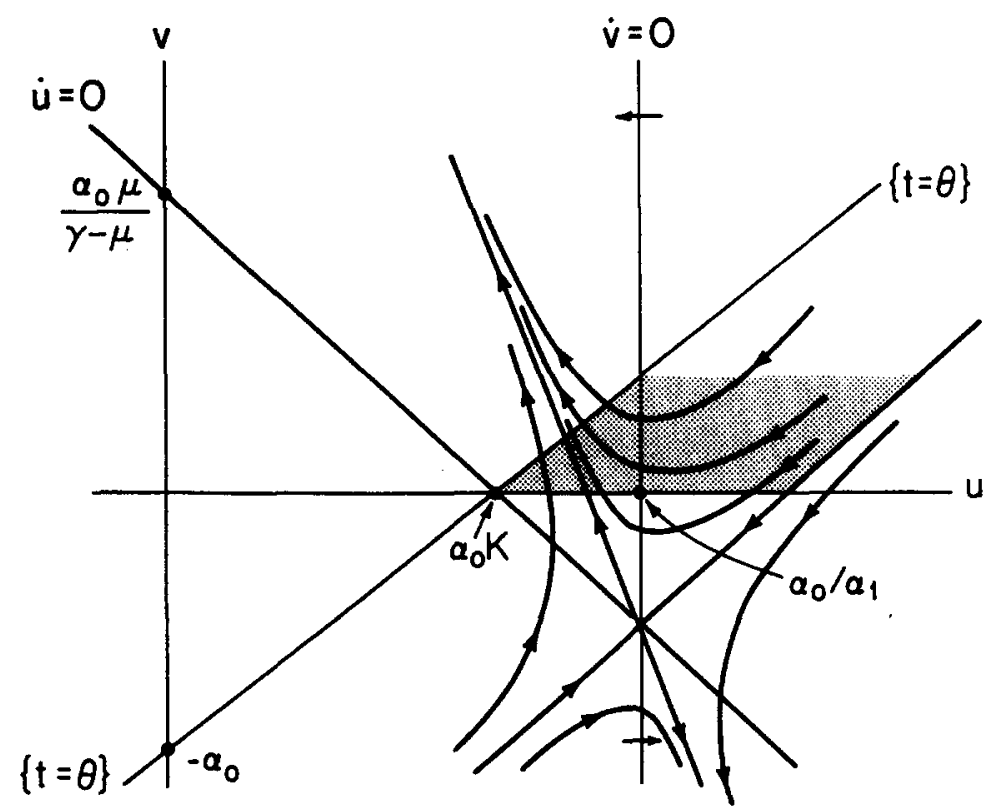

Fig. 8. System (C.3) with $\gamma>\mu$.

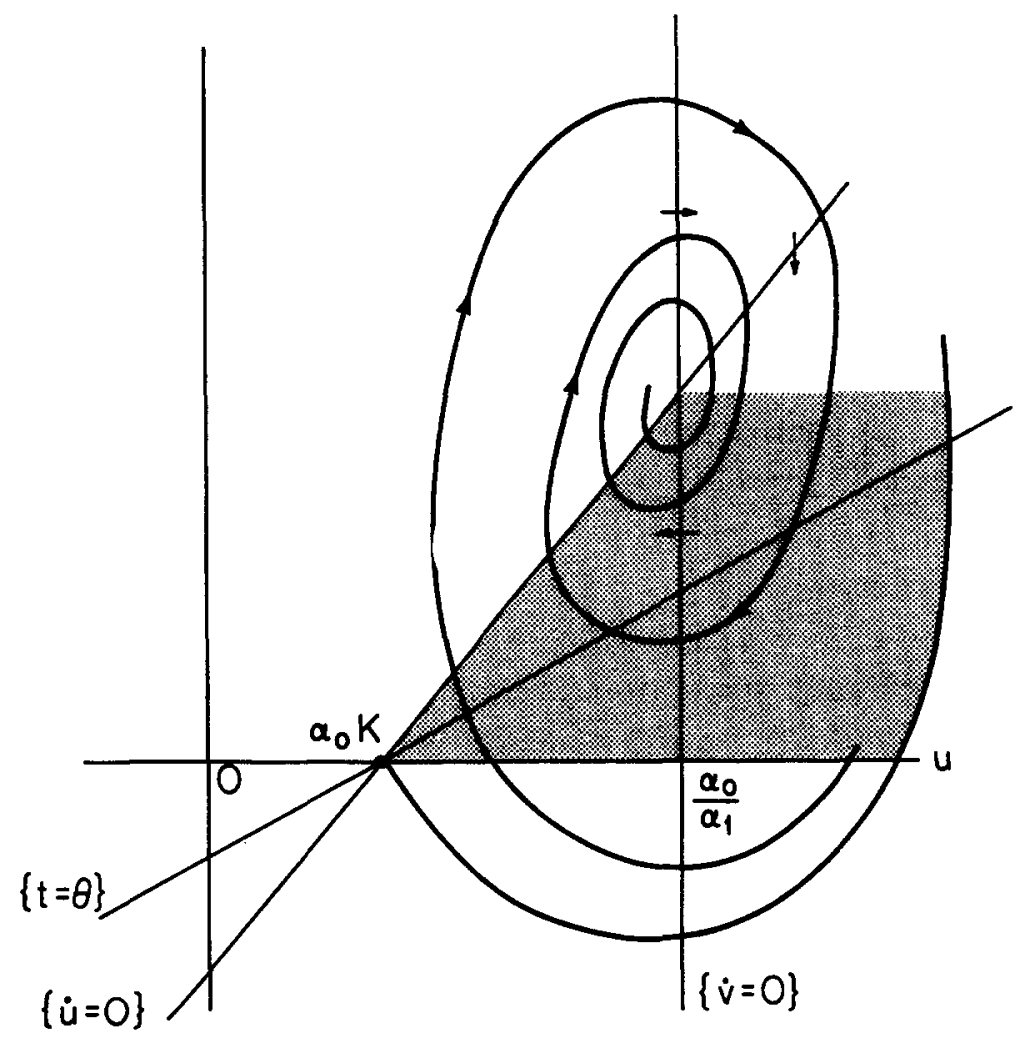

Fig. 9. System (C.3) with $\gamma<\mu$. 
which starts on the horizontal line $v=v(0)$, which ends on the $\{t=\theta\}$-line in the positive quadrant, and which takes exactly $\theta$ units of time to make this trip. One notices that $\dot{u}(\theta)<0$ for such a trajectory and that, except for some situations with very large $\theta$ and large $v(0)$ in the $\gamma<\mu$ case (fig. 10), the solution will have $\dot{u}(t)<0$ for all $t$ between 0 and $\theta$, just as in the Nordhaus formulation.

We now focus on the existence and location of LREP's for this model. By the linearity in eqs. (C.2) and (C.3) and by the general fundamental theory of linear systems of differential equations with constant coefficients, one can show easily that $v(\theta)$ is still a linear function of $v(0)$,

$$
v(\theta)=c_{1} v(0)+c_{2} .
$$

Furthermore, one can use figs. 8 and 9 to see that $c_{1}$ lies between 0 and 1 . For, if $v(0)$ is large positive, most of the business cycle will be in the $i<0$ region and $v(\theta)<v(0)$. On the other hand, if $v(0)$ is large negative, $v(\theta)$ will be $>v(0)$. This implies that there is a $v(0)$ in between for which $v(\theta)=v(0)$ and also that $0<c_{1}<1$ in (C.4). Consequently, just as in the Nordhaus formulation for $\lambda<1$, there is a unique $L R E P$ and any sequence of business cycles [solutions of (C.1) and (C.2)] which satisfies $v_{i+1}(0)=v_{i}(\theta)$ will tend toward this $L R E P$ as $i$ increases.

Finally, we would like to describe the sensitivity of the LREP to changes in the parameters of the model. Recall that the LREP must cross the $\{\dot{v}=0\}$ line and thus must terminate on that segment of the $\{t=\theta\}$-line which lies above the $u$-axis and to the left of the $\{\dot{v}=0\}$-line. Consequently, the $L R E P$ must lie in the regions which are shaded in figs. 8 and 9. If $\theta$ increases, so that one spends more time on the solution curve, then the LREP drops in all three cases - closer to the saddle equilibrium in fig. 8 and further from the sink equilibrium in fig. 9. If $\alpha_{0}$ increases, all the lines move to the right; the shaded region and the $L R E P$ will also move to the right to lower welfare levels. If $\alpha_{1}$ increases, the $\{\dot{v}=0\}$-line will move to the left and so will the $\{t=\theta\}$-line for realistic values of $\alpha_{1}$ and $\beta$ (i.e., $\alpha_{1}^{2} \beta^{2}>1$ ). As a result, the LREP will move down to a higher level of welfare. Putting these effects together, we find that a rise in the natural rate of unemployment $\left(\alpha_{0} / \alpha_{1}\right)$ will entail a higher LREP. In other words, just as in the Nordhaus formulation, the higher the natural rate the more likely it is that continued business cycles will lead to shrinking vote margins and welfare levels.

If $\beta$, the weight on inflation in the vote and welfare function, increases, then $K$ increases and the $\{t=\theta\}$-line swings to the right. If $\mu$ increases and becomes $>\gamma$, the $\{\dot{u}=0\}$-line will turn clockwise about $\left(\alpha_{0} K, 0\right)$ in fig. 9. Both of these effects will move the shaded regions and presumably the LREPs down to higher welfare levels. Finally, increases in $\gamma$ (especially once $\gamma>\mu$ ) appear to have no effect on the $L R E P$. 
One can use the special case where $\mu=\gamma$ to illustrate more clearly the results in this appendix. With a little analysis, one can find tractable analytic solutions to (C.1) for this case. In particular,

$$
\begin{aligned}
& v(\theta)=c_{1} v(0)+c_{2}, \quad \text { wheré } \\
& c_{1}=\left[1+\alpha_{1} \gamma K \theta\left(\left(\mathrm{e}^{\mu \theta}-1\right) / \mu \theta\right)\right]^{-1}, \\
& c_{2}=\alpha_{0} \gamma \theta_{1} /\left(1+\alpha_{1}^{2} \beta\right) .
\end{aligned}
$$

Note that $0<c_{1}<1$ and that the initial $v(0)$ for the $L R E P$ satisfies

$$
v(0)=\left(\alpha_{0} / \alpha_{1}^{2} \beta\right)\left(\mu \theta /\left(\mathrm{e}^{\mu \theta}-1\right) .\right.
$$

In this form, it is straightforward to check that an increase in $\alpha_{1}, \beta, \mu$, or $\theta$ or a decrease in $\alpha_{0}$ will bring $v(0)$ (and hence the $L R E P$ ) down to a higher level of votes and welfare.

\section{Appendix D: A $L R E P$ can have more welfare than Nordhaus' long-run welfare maximizer}

In section 3 of his paper, Nordhaus (1975, p. 175) asked: 'In the absence of political constraints what are the optimal levels of unemployment and inflation?' He chose as his solution the steady state maximizer of (1) subject to the macroeconomic constraints (3) and (4). In the notation of (1), (3), (4), this steady state satisfies

$$
f^{\prime}(u) /(1-\lambda)=-(\rho+\gamma(1-\lambda) /(\rho+\gamma)(1-\lambda))(\partial g / \partial u) /(\partial g / \partial \pi)
$$

and lies on the long-run Phillips curve. So that we can continue our analysis of the previous appendices, we will work in $u-v$ space instead of $u-\pi$ space. Fig. 10 shows the level sets of $g$ (as in fig. 5) and the long-run Phillips curve $(L R P C)$. Point $P_{0}$, where the $L R P C$ is tangent to a level set, represents the 'gold-rule policy' of no-discounting, i.e., $\rho=0$ in (D.1). Point $P_{\infty}$, where the LRPC goes through a peak in a level curve, represents the 'purely myopic policy', i.e., $\rho=\infty$ in (D.1). This is the point which according to Nordhaus (1975, pp. 178-179) will be chosen by a democratic government 'under conditions where voting is an appropriate mechanism for social choice'. The actual welfare maximizer $\boldsymbol{P}_{\rho}, 0<\rho<\infty$, lies somewhere between $\boldsymbol{P}_{0}$ and $\boldsymbol{P}_{\infty}$ on the LRPC. Nordhaus uses the position of $P_{\rho}$ relative to $P_{\infty}$ to draw his basic welfare principle about business cycles. 


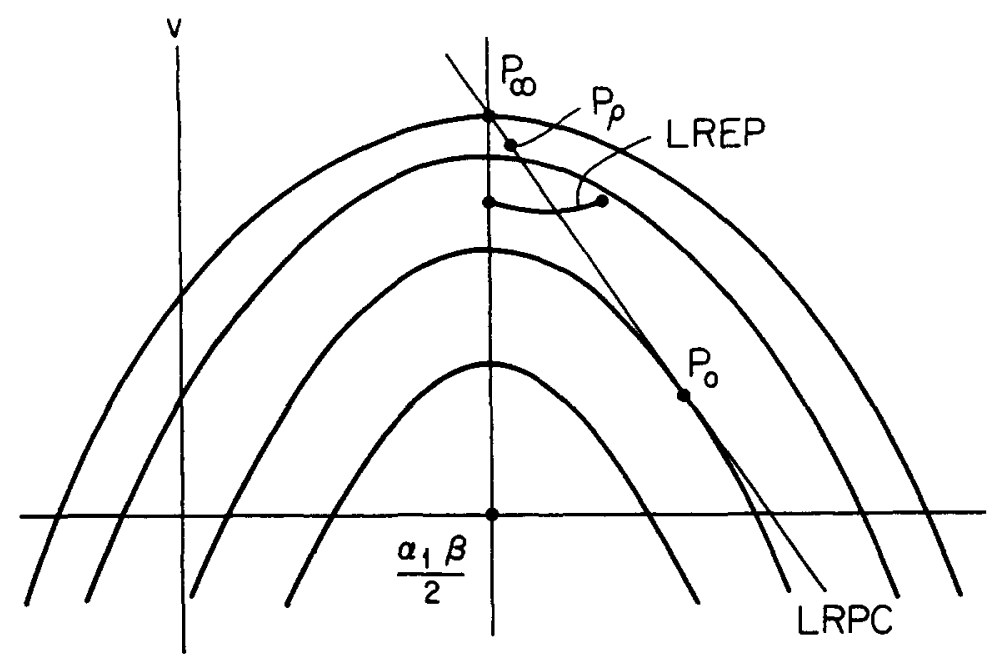

Fig. 10

We will show that a long-run equilibrium path (LREP) can lie on the opposite side of $P_{\rho}$ than $P_{\infty}$ does and that this $L R E P$ can have more welfare at each point on the path than the steady state 'welfare-maximizer' $\boldsymbol{P}_{\rho}$ does. We will use Nordhaus' formulation; in it,

$$
P_{\infty}=\left(\frac{1}{2} \alpha_{1} \beta,\left(\alpha_{0}-\frac{1}{2} \alpha_{1}^{2} \beta\right) /(1-\lambda)\right),
$$

i.e., $P_{\infty}$ lies on the $u(\theta)$-line. Let $(u(t), v(t))$ be a $L R E P, 0 \leqq t \leqq \theta$. By (A.3) and (B.2),

$$
\begin{aligned}
u(\theta) & =\frac{1}{2} \alpha_{1} \beta, \quad u(0)=\left(\frac{1}{2} \alpha_{1} \beta+B / A\right) \mathrm{e}^{-A \theta}-B / A, \\
v(0) & =v(\theta) \\
& =(1 /(1-\lambda))\left\{\alpha_{0}+\alpha_{1}(B / A)+\frac{1}{2}\left(\alpha_{1}^{2} \beta \gamma \lambda / A\right)(k((A+\gamma(1-\lambda)) \theta) / k(\gamma(1-\lambda) \theta))\right.
\end{aligned}
$$

where $k(x) \equiv\left(1-\mathrm{e}^{-x}\right) / x$, still.

Note that as $\theta \rightarrow 0$,

$$
(u(0), v(0)) \rightarrow P_{\infty} \quad \text { and } \quad(u(\theta), v(\theta)) \rightarrow P_{\infty} .
$$

Now fix all the parameters except $\theta$. We want to trace out the locus of the $(u(0), v(0))$ endpoints of our $L R E P$ as $\theta$ increases from 0 . This locus slopes downward and to the right with a non-zero derivative; for, by (D.2),

$$
\left.(\mathrm{d} / \mathrm{d} \theta) u(0)\right|_{\theta=0}=\left(\frac{1}{2} \alpha_{1} \beta+B / A\right)(-A)=\frac{1}{2} \alpha_{1} \beta \gamma \lambda>0,
$$

and by (D.3),

$$
\left.(\mathrm{d} / \mathrm{d} \theta)(v(0))\right|_{\theta=0}=\frac{1}{2}\left(\alpha_{1}^{2} \beta \gamma \lambda /(1-\lambda)\right)\left(k^{\prime}(0) / k(0)\right)<0,
$$

since $k(0)=1$ and $k^{\prime}(0)=-\frac{1}{2}$. 
Since the tangent to the level set of $g$ is horizontal at $\boldsymbol{P}_{\infty}$,

$$
g(u(0), v(0))>g\left(P_{\infty}\right) \text { for } \theta \text { small. }
$$

For such $\theta$,

$$
g(u(t), v(t))>g\left(P_{\infty}\right) \text { for } \quad 0 \leqq t \leqq \theta,
$$

because the LREP is u-shaped and $g$ is concave. As $\rho \rightarrow \infty, P_{e} \rightarrow P_{\infty}$. Therefore, for $\rho$ big enough and $\theta$ small enough, the welfare at each point along the $L R E P$ will exceed the welfare at the steady state $P_{\rho}$.

\section{References}

Beck, N., 1982, Does there exist a political business cycle: A Box-Tiao analysis, Public Choice $38,205-209$.

Chappell, H.W., Jr. and W.R. Keech, 1983, Welfare consequences of the six-year presidential term evaluated in the context of a model of the U.S. Economy, American Political Science Review 77, 75-91.

Clark, C.W., 1976, Mathematical bioeconomics (Wiley Interscience, New York).

Fair, R.C., 1978, The effect of economic events on votes for president, Review of Economics and Statistics 60, 159-173.

Frey, B.S. and H.J. Ramser, 1976, The political business cycle: A comment, Review of Economic Studies 43, 553-556.

Golden, D.G. and J.M. Poterba, 1980, The price of popularity: The political business cycle reexamined, American Journal of Political Science 24, 696-714.

Hibbs, D.A., Jr., 1982, The dynamics of political support for American presidents among occupational and partisan groups, American Journal of Political Science 26, 312-332.

Keech, W.R., 1980, Elections and macroeconomic policy optimization, American Journal of Political Science 24, 345-367.

Keech, W.R. and C.P. Simon, 1983, Inflation, employment, and electoral terms: When can reform of political institutions improve macroeconomic policy?, in: K. Monroe, ed., The political process and economic change (Agathon, New York) 77-107.

Kramer, G.H., 1971, Short-term fluctuations in U.S. voting behavior, 1896-1964, American Political Science Review 65, 131-143.

Lächler, U., 1978, The political business cycle: A complementary study, Review of Economic Studies 45, 369-375.

Lindbeck, A., 1976, Stabilization policies in open economics with endogenous politicians, American Economic Review 66, 1-19.

McCallum, B.T., 1978, The political business cycle: An empirical test, Southern Economic Journal 44, 504-515.

MacRae, C.D., 1977, A political model of the business cycle, Journal of Political Economy 85, 239-263.

Maloney, K.J. and M.L. Smirlock, 1981, Business cycles and the political process, Southern Economic Journal 48, 377-392.

Monroe, K.R., 1983, Political manipulation of the economy: A closer look at political business cycles, Presidential Studies Quarterly 13, 37-49.

Nordhaus, W.D., 1975, The political business cycle, Review of Economic Studies 42, 169-190.

Paldam, M., 1979, Is there an electional cycle?: A comparative study of national accounts, Scandinavian Journal of Economics 81, 323-342.

Paldam, M., 1981, An essay on the rationality of economic policy: The test case of the electional cycle, Public Choice 37, 287-305.

Tufte, E.R., 1978, Political control of the economy (Yale University Press, New Haven, CT). 